\title{
Palliative care in emergency medicine: A perennial skill with a new emphasis
}

\author{
Jennifer Hughes, MD, CCFP (EM) (PC)*
}

The emergency department (ED) is an important but underrecognized setting for the provision of palliative care. Emergency medicine physicians provide care daily for people living with advanced cancer and other incurable illnesses such as congestive heart failure, chronic obstructive lung disease, end-stage renal disease, and dementia. In an Ontario study, $83 \%$ of patients who died of cancer visited an ED at least twice in the last 6 months of life. ${ }^{1}$ The number of deaths from these illnesses is expected to grow with our aging population. Furthermore, these patients often present to the ED in crisis with uncontrolled symptoms or a terminal event. ${ }^{2}$ These patients constitute a large part of ED visits, yet their care is arguably outside of the traditional norms and goals of emergency medicine, which are focused on resuscitation and life prolongation. They are often given a less intense focus, and their complex needs are difficult and sometimes uncomfortable for emergency medicine physicians to address. Advanced palliative care competencies should thus be part of the emergency physician's skill set, in the same manner as are advanced airway management and bedside ultrasonography.,

Emergency physicians provide care frequently to patients in a palliative context. Therefore we must ask how comfortable are we with this skill set? What formal education are we receiving? The two papers published in this edition help us answer these questions.

Dr. Argintaru and colleagues highlight the unique challenges that emergency physicians face when having goals of care conversations. ${ }^{5}$ Although many of the barriers seem obvious, such as limited information and limited time, others may require deeper probing, such as exploring the responsibility for these conversations. The majority of ED physicians felt that these discussions were within their scope, and three-quarters felt that they had been adequately trained to have them. Yet, over half felt that the admitting service was most appropriate to have these discussions. It is interesting to note that $55 \%$ of respondents felt that a 24 -hour palliative care service would facilitate the ability to conduct goals of care conversations in the ED, but only $6 \%$ felt that palliative care should be specifically consulted. There are several conflicting messages in these data that require careful parsing. Despite this ambiguity, it is imperative that we, as ED physicians, take ownership of these conversations with our patients when we can. In many instances, a well-executed 5-minute conversation with a patient or substitute decision maker can significantly alter the trajectory of patient care. ${ }^{6}$

Dr. Baylis and colleagues provide a valuable understanding of the educational experiences of Canadian emergency physicians and resident physicians. ${ }^{7}$ Just one of 26 residency programs surveyed has a mandatory palliative medicine rotation, and only $38.5 \%$ have a lecture or seminar on palliative medicine at all. In practice, these lectures are often done on an ad hoc basis when local expertise exists, as opposed to having a curriculum that meets predetermined learning objectives. The Royal College identifies "providing end-of-life care" as a core competency, yet does not include other equally important palliative care skills, such as symptom management, goals of care conversations, and communicating prognosis. The College of Family Physicians of Canada has no palliative medicine competencies listed for the Certification of the College of Family Physicians in Emergency Medicine, CCFP (EM), residents. Although most CCFP (EM) residents would have exposure to a palliative care rotation through family medicine residency

From the ${ }^{*}$ Cumming School of Medicine, University of Calgary, Calgary, AB.

Correspondence to: Dr. Jennifer Hughes, Foothills Medical Centre, 140329 St NW, Room C231, Calgary, Alberta T2N 2T9; Email: Jennifer.Hughes@ AHS.ca

(c) Canadian Association of Emergency Physicians

CJEM 2019;21(2):163-164

DOI 10.1017/cem.2019.7 
training, Dr. Baylis rightly questions the relevance of that experience to the ED where time is limited and decision-making is often precipitated by crises.

Emergency physicians will likely encounter far more palliative care situations than thoracotomies during their careers. Quality teaching of core palliative care domains in emergency medicine should be fulsome and mandatory. Education in Palliative and End-of-life Care for Emergency Medicine (EPEC-EM) is an American program designed to educate physicians on the essential palliative clinical competencies in the ED. ${ }^{8}$ There is great potential to use and adapt this curriculum to train Canadian residents and physicians. Furthermore, Pallium Canada is in the process of developing an interdisciplinary 1-day course in palliative care for emergency medicine providers.

The concept of palliative care is relatively new in emergency medical education. In the United States, it is a recognized subspecialty of emergency medicine. ${ }^{8}$ In Canada, there are few dually certified emergency and palliative care physicians to provide expertise and leadership. We need more physicians pursuing training in palliative care to advance research and teaching.

Palliative care has always been an important part of the care provided by emergency physicians. Despite improvements in advanced care planning, there will always be some patients who present in crisis or who will change their goals as they move through their disease trajectory. Emergency physicians are skilled innovators who think outside of the box to adapt to changing environmental and patient needs. We must now turn our creativity to find solutions to provide excellence in palliative care within our unique setting. It is time to recognize, formalize, and improve our role as palliative care providers in the ED.

Keywords: Palliative care, emergency medicine, medical education

Competing interests: None declared.

\section{REFERENCES}

1. Barbera L, Taylor C. Why do patients with cancer visit the ED near the end of life? CMA7 2010;182 (6):563-8.

2. Grudzen CR, Richardson LD, Morrison M, et al. Palliative care needs of seriously ill, older adults presenting to the emergency department. Acad Emerg Med 2010;17:1253-7.

3. Gisondi M. A case for education in palliative and end-of-life care in emergency medicine. Acad Emerg Med 2009;16(2): 181-3.

4. Frist $W$, Presley $M$. Training the next generation of doctors in palliative care is the key to the new era of value-based care. Acad Med 2015;90(3):1-4.

5. Argintaru N, et al. Perceived barriers and facilitators to goals of care discussions in the emergency department: A descriptive analysis of the views of emergency medicine physicians and residents. CFEM 2019;21(2):211-218.

6. Wang D. Beyond code status: palliative care begins in the emergency department. Ann Emerg Med 2017;69:437-43.

7. Baylis $\mathrm{J}$ et al. Palliative and end-of-life care education in Canadian emergency medicine residency programs: A national cross-sectional survey. C7EM 2019;21(2):219-225.

8. Emanuel LL, Quest T. The education in palliative and end of life care for emergency medicine (EPEC-EM). The EPEC project; 2008. Available at: http://www.epec.net/EPEC/Webpages/epecem.cfm (accessed December 10, 2015). 